Structured Variation in Parental Beliefs about Autism

\author{
Jane D. McLeod \\ Indiana University \\ Lydia DiSabatino
}

Chicago Public Schools

\begin{abstract}
Please direct all correspondence to Jane D. McLeod, Department of Sociology, Indiana University, Ballantine Hall 702, 1020 E. Kirkwood Avenue, Bloomington, IN 47405. email:

jmcleod@indiana.edu
\end{abstract}

\author{
Word count: 9,101 \\ Number of tables: 6 \\ Number of figures: 1 \\ Running head: Parental beliefs about autism
}


We used data from the 2011 Survey of Pathways to Diagnosis and Services $(N=1,420)$ to evaluate a conceptual model linking social background (race/ethnicity, socioeconomic status) to parental distress through children's clinical profiles and parental beliefs about the nature and causes of their child's autism. Children's clinical profiles varied by social background; white children and children of more highly educated and affluent parents were less likely to experience comorbid conditions and were more likely to be diagnosed with Asperger's. Parental beliefs also varied such that parents of racial/ethnic minority children and parents of lower SES perceived their child's condition as more uncertain, and were less likely to attribute it to genetic causes. Parents of Hispanic children and with lower incomes were more likely to be upset by the child's condition. Although parental beliefs had independent associations with distress, children's clinical profiles contributed more to explaining variation in distress.

KEYWORDS: autism, stress, illness beliefs, children, parents 


\section{Structured Variation in Parental Beliefs about Autism}

Stress researchers assert that responses to stressors depend on their meanings to the person (Lazarus and Folkman 1984; Pearlin 1989; Thoits 1995). Psychological stress researchers have advanced this assertion through analyses of stress appraisals (e.g., Folkman et al. 1996; Cohen, Kamarck, and Mermelstein 1983), while sociological stress researchers have analyzed responses to objective stressors based on contextual indicators of meaning (e.g., beliefs and values; identity relevance; prior role experience; e.g., Simon 1995; Thoits 1991; Wheaton 1990). Neither set of researchers has investigated whether the meanings of stressors vary systematically across major social groups, such as those defined by race/ethnicity and socioeconomic status. Analyses of structured variation in meaning complement analyses of variation in stress exposure and vulnerability (e.g., Turner, Wheaton, and Lloyd 1995) by identifying another path through which social arrangements shape the stress process (McLeod 2012).

We take a first step toward understanding structured variation in the meanings of stressful life experiences by analyzing socioeconomic and racial/ethnic variation in parental beliefs about the nature and causes of autism and the implications of that variation for parental distress. Autism lends itself to this analysis because raising a child with autism is a potentially stressful experience for which variation in parental beliefs and parental responses has been observed (Benson 2010; Goin-Kochel and Myers 2005; Hartley, Schaidle, and Burnson 2013). We situate our analysis in a conceptual model that links social background to parental distress through the child's clinical profile and parental beliefs. 


\section{BACKGROUND}

Figure 1 depicts our conceptual model. We propose that children's social backgrounds are linked to parental distress through the associations of: (a) social background with children's clinical profiles; (b) (c) social background and children's clinical profiles with parental beliefs; and (d) (e) children's clinical profiles and parental beliefs with parental distress. In the language of the stress process, we treat children's clinical profiles as objective stressors and parental beliefs as the meanings parents assign to them.

<Figure 1 about here>

\section{Variation in clinical profiles}

Children's clinical profiles may vary by socioeconomic status (SES) and race/ethnicity for at least two reasons: differential diagnosis and differential access to care. With respect to diagnosis, children from socioeconomically disadvantaged backgrounds are less likely to receive autism diagnoses and, when they do, are diagnosed at later ages and with more severe conditions than children from advantaged families (e.g., Fountain, King, and Bearman, 2011; Rosenberg et al. 2009). The same holds true for racial and ethnic minority children, who are more likely than white children to receive diagnoses other than autism first and whose autism diagnosis is, as a result, delayed (Mandell et al. 2007). Whether variation in clinical profiles reflects differences in symptom presentation, parents' descriptions of symptoms, or provider bias remains unclear (Cuccaro et al. 1996; Mandell et al. 2007). Regardless of the reason, structured variation in diagnosis implies that racial and ethnic minority children and children from lower socioeconomic backgrounds who have received autism diagnoses will have more severe symptoms than other children who have received diagnoses. 
Alternatively, and relatedly, variation in clinical profiles may be attributable to differential access to treatment. Autism responds to early treatment (National Research Council 2001), and children who are diagnosed at later ages are less likely to receive early intervention. The uncertain effectiveness of specific treatments for specific children compounds the disadvantage. Many parents of children with autism report trying multiple treatments before identifying one that addresses their child's symptoms (Goin-Kochel, Myers, and Mackintosh 2007). Parents with limited resources may be less able to experiment with treatment alternatives, leaving their children with more severe limitations.

We propose that children's clinical profiles mediate the associations of SES and race/ethnicity with parental distress. Children's symptoms and behaviors influence the day-today realities of caring for the child as well as parental perceptions of stress (Ingersoll and Hambrick 2011; McStay et al. 2014). By extension, structured variation in children's clinical profiles may explain structured variation in parental distress. According to our model, children's clinical profiles may also influence parental beliefs.

\section{Variation in parental beliefs}

We emphasize two components of parental beliefs: beliefs about the nature of the child's condition and causal attributions. With respect to the former, we focus on uncertainty, chronicity, and controllability. With respect to the latter, we focus on genetic and environmental attributions. These features of parental beliefs map closely onto established dimensions of stressful experiences.

The nature of the condition. Uncertainty is central to the experience of stress (Folkman 2010). In experimental settings, uncertainty about when an adverse event, such as an electric shock, will occur is associated with increased physiological indicators of stress (Monat, Averill, 
and Lazarus 1972). Uncertainty is also associated with poor adjustment among people with chronic illness (e.g., Mullins et al. 2017; Santacroce 2003). The parents included in our study reported whether they consider their child's condition "a mystery" and whether it "comes and goes," which we treat as indicators of uncertainty.

The concepts of chronicity and controllability align with stress appraisals. According to Lazarus and Folkman (1984), when faced with a stressor, people begin by appraising how much the situation threatens their well-being (i.e., primary appraisals) and, then, what the options are for coping with it (i.e., secondary appraisals). Chronicity and controllability are among the most studied situational determinants of primary stress appraisals (Cohen, Kessler, and Gordon 1997). Relevant to these concepts, parents in the current study reported whether they believe their child's condition to be lifelong, that the associated problems can be prevented or decreased with treatment, and that they have the power to change the child's condition.

Some evidence suggests that stress appraisals may be socially structured. People in lower socioeconomic positions and racial/ethnic minorities interpret potentially stressful situations as more threatening (Chen et al. 2004) and perceive themselves as less in control of their lives (Mirowsky and Ross 1986) than others. Accordingly, we expect parents of lower socioeconomic status to perceive their children's autism as less certain, more chronic, and less controllable.

Empirical evidence provides some support for this expectation. Using the same data we use here, Zuckerman and colleagues (2015) evaluated variation in parental beliefs about the nature of their child's autism by race/ethnicity and socioeconomic status. They observed that parents with lower educational attainment and who had lower income were more likely to report believing that their child's condition was a mystery. Parents of minority children were less likely to see the 
child's condition as lifelong and household income was inversely associated with parents' believing that they had the power to change their child's condition.

We extend Zuckerman et al.'s analysis by including indicators of the child's clinical profile in the model. Specifically, we evaluate whether children's clinical profiles are associated with parental beliefs and whether variation in clinical profiles accounts for sociodemographic variation in parental beliefs. Parents whose children receive more severe diagnoses (i.e., Autistic Disorder rather than Pervasive Developmental Disorder-Not Otherwise Specified (PDD-NOS) or Asperger's Syndrome) may be more likely to believe that their children's conditions are chronic and less likely to believe they are controllable than parents of children who receive less severe diagnoses. Parents whose children receive diagnoses at later ages may also be more likely to believe that their child's condition is chronic (Hartley et al. 2013).

Causal attributions. Parents encounter competing discourses about the causes of, and appropriate treatments for, autism (Singh 2016). Genetics and genomics frames—which seek causes, treatments, and cures for autism through genetics or genomics research—dominate research and have received strong support from parent advocacy groups. But other frames have also been advanced, including those that locate the causes of autism in post-birth or in utero exposures to toxins, pesticides, or vaccinations.

Within the range of available interpretive frames, parents affirm diverse attributions, including heredity, birth-related trauma, early childhood exposures, and childhood vaccinations (e.g., Al Anbar et al. 2010; Dale, Jahoda, and Knott 2006; Hebert and Koutouglioti 2010). Most existing studies of parental attributions involve small, group-specific samples and do not allow for generalizable comparisons across groups (e.g., Hartley et al. 2013). For example, Latino parents who participated in a small, qualitative interview study attributed the characteristic 
symptoms of autism to disrupted family relations (Zuckerman et al. 2014). Without a comparison to other groups, we cannot know whether the views expressed by these parents are unique.

As was true for parental beliefs about the nature of the child's condition, Zuckerman and colleagues (2016) presented the only nationally-representative data on parental causal attributions. Beliefs about genetic causes were most common but a sizeable minority of parents attributed their child's condition to in utero or post birth exposures. Causal attributions differed by SES and race/ethnicity, with Hispanic parents less likely to attribute their child's condition to genetics or heredity and low-income parents more likely to attribute it to environmental exposures.

Zuckerman's findings accord with prior research on racial/ethnic and socioeconomic differences in parental beliefs about mental health problems. In a sample of parents of elementary school children who qualified for special education services, African American parents were less likely to attribute ADHD to genetic origins, less likely to use medical labels to refer to their children's condition, and more likely to attribute ADHD to excessive sugar intake (Bussing, Schoenberg, and Perwien 1998). Similarly, Yeh and colleagues (2004) observed that parents of African American and Latino youth with mental health problems were less likely than whites to endorse "biopsychosocial causes" (e.g., physical causes, trauma).

In our analysis, we evaluate whether the child's clinical profile influences attributions and explains sociodemographic variation. There is some evidence, for example, that parents whose children were diagnosed at younger ages are more likely to affirm genetic explanations (GoinKochel and Myers 2005).

\section{Parental beliefs and distress}


As components of the meanings parents give to their child's condition, parental beliefs about the nature and causes of autism may also influence their responses. Previous studies present mixed evidence regarding the relevance of parental beliefs for one type of response: treatmentseeking. Parents who believe that their child's condition was caused by environmental factors report seeking nutritional and detoxification treatments (Al Anbar et al. 2010). Parents who believe their child's autism can be cured prefer programs that have cure as a goal (Hebert and Koulouglioti 2010). However, using the same nationally-representative data we use in our analysis, Zuckerman and colleagues (2015) observed mostly nonsignificant associations of beliefs with medication use, use of behavioral therapies, and use of complementary or alternative therapies.

We consider the implications of parental beliefs for a different response: parental distress. We hypothesize that parental beliefs about the nature and causes of their child's condition influence parental distress and mediate the associations of race/ethnicity and SES with distress.

Parents of children with autism report higher levels of stress and distress than parents of typically developing children and of children with other developmental disabilities and chronic health conditions (see review in Serrata 2012). Little evidence is available about structured variation in parental distress. Bishop and colleagues (2007) found that African American parents reported less negative impact of their child's autism than White parents. Magaña and Smith (2006) found that caregiving responsibilities increased depression among Latino parents of children with developmental disabilities but not African American parents. If these studies generalize, we would expect Hispanic parents to report higher levels of distress than white and African American parents. 
Parental beliefs may also influence distress. Regarding the nature of the condition, studies show consistently that stressors that are appraised as chronic and uncontrollable increase negative affect, reduce positive affect (e.g., Blaxton and Bergeman 2017; Gannon and Pardie 1989), and increase feelings of helplessness and low expectations for the future (Weiner 1985). Among parents of children with disabilities, the degree to which the disability is perceived as comprehensible and predictable moderates parental stress and increases well-being (Kimura and Yamazaki 2016). Accordingly, we expect that parents who perceive their child's condition as uncertain, chronic, and uncontrollable will report more distress than other parents.

The limited empirical evidence is consistent with this expectation. In a study of U.S. parents whose children were receiving treatment for autism, parents who believed that their child's behavior problems were more stable and that they themselves were less able to control the problems reported higher levels of parenting burden (Hartley et al. 2013). Similarly, Falk and colleagues (2014) reported that parents of children with autism who perceived that they had influence over their child's development reported less depression and stress than other parents.

Causal attributions may also influence parental distress through their associations with feelings of guilt and blame. Qualitative research on parental adjustment to a child's autism documents the prominence of these emotions. Mothers report wondering whether they inadvertently contributed to their child's condition and blame themselves for choices they made during pregnancy or after birth (e.g., DePape and Lindsay 2015). Mothers who blame themselves or report feeling guilty have lower levels of self-efficacy when coping with their child's autism (Francis 2013; Kuhn and Carter 2006) and report higher levels of depression, anxiety, and distress (Foody et al. 2014). Genetic attributions could lessen guilt and blame by absolving parents of personal responsibility for their child's condition and encouraging empathy from 
others. However, contrary to this prediction, Dale et al. (2006) found that some parents blame themselves for passing along genetic vulnerability to their children (see also Phelan 2005).

To summarize, in this analysis we evaluate a conceptual model that links children's social backgrounds and clinical profiles to parental beliefs about autism and parental distress. We ask the following specific research questions:

- Do parental beliefs about the nature and causes of a child's autism differ by race/ethnicity and socioeconomic status? To what extent can observed differences be explained by differences in the child's clinical profile?

- Does parental distress differ by race/ethnicity and socioeconomic status? To what extent are the differences attributable to differences in the child's clinical profile? To parental beliefs?

The answers to these questions contribute to specific knowledge about parents' experiences of autism and also to general knowledge about the role of meaning in the stress process.

\section{METHODS}

\section{Data}

Data for the analysis are from the 2011 Survey of Pathways to Diagnosis and Services. The Pathways survey is a follow-up to the 2009-2010 National Survey of Children with Special Health Care Needs (NS-CSHCN) which is a module of the National Center for Health Statistics' State and Local Area Integrated Telephone Survey (SLAITS) Program (Bramlett et al. 2014). The sample for the NS-CSHCN was designed to yield 750 interviews with parents or guardians of children with special health care needs in each state. To achieve this goal, the NS-CSHCN screened households to identify those that had at least one child aged 0 to 17 with special health care needs. Children were identified as having special health care needs if their parent: (1) 
responded affirmatively to at least one of five stem questions on special health needs (e.g., "Does your child currently need or use medicine prescribed by a doctor, other than vitamins?’); (2) reported that the health care needs resulted from a health condition; and (3) that the condition had lasted or was expected to last 12 months or longer. Respondents who had at least one qualifying child answered a series of more detailed questions about the child's health care. In 2009-2010, 40,242 households completed the NS-CSHCN interview.

The Pathways sample was drawn from the households that completed the NS-CSHCN. Households were eligible for the Pathways survey if: (1) a doctor or other health care provider ever told the parent that the child had "Autism, Asperger's Disorder, pervasive developmental disorder, or other autism spectrum disorder," "any developmental delay that affects [his/her] ability to learn," and/or "intellectual disability or mental retardation," and (2) the child would be between the ages of 6 and 17 at the time of the Pathways interview $(\mathrm{N}=7,572)$. A random sample of 6,090 eligible children was selected for re-contact. Telephone interviews were conducted with whichever parent or guardian completed the NS-CSHCN survey: $80 \%$ were the mother, $14 \%$ the father, and 6\% another relative or guardian. Interviews were completed with 4,032 respondents. For brevity's sake, we refer to all respondents as "parents." Our analysis focused on children whose parent reported that they currently had an autism diagnosis $(\mathrm{N}=1,420)$. Of the 1,420 respondents, 1,092 were mothers, 250 were fathers, and 78 were another relative or guardian. We reran all models in the sample of parents and observed virtually identical results.

\section{Measures}

Our analysis incorporates three sets of dependent variables: beliefs about the nature of the child's condition; beliefs about its causes; and distress. 
Beliefs about the nature of the condition. The variables for parental beliefs were adapted from the Revised Illness Perception Questionnaire (Al Anbar et al. 2010). The stem question for this series, and for questions about causal attributions, was "Would you say you definitely agree, somewhat agree, somewhat disagree, or definitely disagree with these statements about [name's] learning and developmental conditions."

Parents answered five relevant questions:

- $\{$ Name]'s condition is a mystery to me (uncertainty).

- $\{$ Name]'s symptoms come and go (uncertainty).

- $\{$ Name\}'s condition is likely to be lifelong rather than temporary (chronicity).

- The problems related to [name]'s condition can be prevented or decreased with treatment (controllability).

- I have the power to change [name]'s condition (controllability).

Table 1 presents the frequency distributions for these variables. Less than half of the parents saw the child's condition as a mystery ( $42.41 \%$ definitely or somewhat agree) and the symptoms as coming and going (29.70\% definitely or somewhat agree). Almost all of the parents definitely or somewhat agreed that the child's condition is lifelong and that treatment can help (94.62\% and $80.32 \%$, respectively). Few parents definitely agreed that they have the power to change the condition (10.10\%) although over 40\% (42.43\%) had some faith that they could do so. We created binary variables to distinguish parents who "definitely agreed" from all others. We reestimated all models using the original ordinal variables to evaluate the sensitivity of the results to this coding decision. In no case did the substantive conclusions change.

The stem question is not specific to autism, which could raise concerns about whether parental beliefs pertain specifically to autism. Although a potential limitation, the generality of 
the question reflects the reality that children with autism often experience comorbid intellectual, developmental, and mental health problems (Rosenberg et al. 2011), all of which are likely to influence parental beliefs. By including measures of comorbid conditions in our models, we can determine whether parents' views differ systematically depending on these other conditions.

$<$ Table 1 about here>

Beliefs about causes. These variables came from the same set of questions, three of which addressed causes:

- I think [name]'s condition is genetic or hereditary.

- I think [name]'s condition was caused by something [he/she] was exposed to in utero, that is, before [he/she] was born.

- I think [name]'s condition was caused by something [he/she] was exposed to after [he/she] was born.

In addition, parents were asked:

- Has [name] experienced any accident, injury, or illness that you feel has had an effect on [his/her] behavior or development?

For purposes of the analysis, we created binary variables for affirming a genetic cause (definitely agreed) and affirming an environmental cause (definitely agreed that in utero exposures, post-birth exposures, or accident/injury/illness were a cause). Table 1 presents the frequency distributions for these variables. Most parents of children in this sample believed that their child's condition was genetic (68.55\% definitely or somewhat agreed). A smaller proportion believed that the child's condition had an environmental cause. As for parental beliefs about the nature of the condition, we re-estimated all models using the original ordinal variables with no substantive change in conclusions. 
Parental distress. Our measure of parental distress was based on a single agree-disagree question, with frequencies in Table 1, "When I think about [name]'s condition, I get upset." We created a binary variable to distinguish parents who "definitely" agreed from all others but also re-estimated the models using the original, ordinal variable. Although we would have a preferred a more robust measure of distress, this is the only relevant item in the data set. Despite its limitation, it demonstrates meaningful group variation.

Social background. We predicted parental distress and beliefs from three indicators of the child's social background — race/ethnicity, parental education, and household income — as well as from the child's clinical profile and other sociodemographic controls. To protect the confidentiality of survey respondents, the NCHS "coarsened" many of the independent variables by collapsing response categories. For example, the child's race/ethnicity is available only in four categories to reduce the risk that specific children from small sampling areas can be identified. As a result, there is less variation in some of the predictor variables than was originally reported by parents.

Race/ethnicity was coded based on the parent's response to a question about whether the child is of Hispanic origin and about which category(ies) best describes the child's race. The data set includes a summary variable for race/ethnicity that indicates whether the child is Hispanic, non-Hispanic white, non-Hispanic Black, or non-Hispanic other (Asian, American Indian or Alaska Native, Native Hawaiian or Pacific Islander, and those who identify with multiple races). Consistent with what we know about the diagnosed prevalence of autism spectrum disorders, most respondents (75.04\%) were white (Newschaffer et al. 2007). We included two indicators of parental SES. The first, parental education, is a binary variable for whether the parent had more than a high school education. Most respondents $(86.07 \%)$ had a high level of education 
according to this indicator. This is a higher percentage than we would expect (roughly $40 \%$ of adults 25-54 years of age had a high school education or less in 2011), indicating that the sample is skewed toward highly educated respondents. The second, household income, was measured as a percentage of the federal poverty level for a household of that size, available in the data set as four dummy variables: 0-99\% of the federal poverty level, 100-199\%, 200-399\%, and 400\% and higher. The sample is skewed toward higher incomes but with reasonable numbers of lowincome respondents.

Because the demographic questions appeared toward the end of the NS-CSHCN, the rate of missing data for these variables was higher than for other items. SLAITS staff imputed missing values (Centers for Disease Control 2012). Four of the variables we use here include singly imputed values: child's race, ethnicity, highest parental education, and household income. Of the 1,420 children represented in our analysis sample, 6 have imputed values for race, 3 for Hispanic ethnicity, 6 for highest education level of any parent in household, and 188 for household income.

The child's clinical profile and control variables. We included several indicators of the child's clinical profile. These include the age at which the child was diagnosed, current diagnosis of developmental delay, current diagnosis of intellectual disability, specific autism diagnosis (pervasive developmental disorder, autistic disorder, Asperger's syndrome, multiple diagnoses, or not known), whether the child had comorbid emotional and behavioral problems (based on a high score on the Strengths and Difficulties Questionnaire, SDQ; Goodman 1997), ${ }^{1}$ and the level of functional limitations. The SDQ is a 5-item scale assessing conduct problems, hyperactivity, emotional symptoms, peer problems, and pro-social behavior. A high score is defined as a score of 6 or above on a 10-point scale. We coded functional limitations as a binary variable (1=yes, 
$0=$ no) based on three questions from the NS-CSHCN screener: "Is your child/Are any of your children limited or prevented in any way in [his/her/their] ability to do the things most children of the same age do?" "Is [child's \} limitation in abilities because of ANY medical, behavioral, or health condition?" "Is this a condition that has lasted or is expected to last 12 months or longer?" Children whose parents responded yes to all three questions were coded as having functional limitations.

The frequencies for these variables are given in Table 1. Over half the children in this sample were diagnosed before age $6(57.40 \%)$ and almost half $(42.67 \%)$ had received more than one diagnosis at some time. Among those with a single diagnosis, Asperger's syndrome was most common.

In all models, we controlled the child's gender and current age in years. Consistent with national prevalence estimates, most of the children were male (Newschaffer et al. 2007). Agethe only continuously coded variable — had a mean of 11.51 years (not in table).

\section{Analytic strategy}

We used logistic regression models to analyze the predictors of our outcomes. The first two sets of models (Tables 2 and 3) predicted parental beliefs about the nature of the condition and causal attributions from race/ethnicity, SES, the child's clinical profile, and controls. The second set of models (Table 4) paralleled those but with parental distress as the dependent variable. We estimated a final model that evaluated the degree to which parental beliefs explained structured variation in parental distress.

Of the 1,420 children eligible for the analysis, 149 had missing values on one or more variables $(\mathrm{N}=128$ missing on one variable; 14 missing on two variables; 7 missing on three or more variables). The rate of missing data was similar across racial/ethnic groups (from $6.74 \%$ for 
Blacks to $13.51 \%$ for "other" races), and groups defined by parental education $(10.41 \%-11.00 \%)$ and income $(9.36 \%-12.63 \%)$. The question about genetic causation had the highest rate of missing data $(\mathrm{N}=81)$. The remaining missing cases were scattered among the variables for the nature of the condition (from $\mathrm{N}=3-20)$, the SDQ $(\mathrm{N}=19)$, and functional limitations $(\mathrm{N}=11)$. To preserve cases, we estimated models using all cases with valid values on each specific dependent variable.

To facilitate interpretation, we present average marginal effects, which can be interpreted as the predicted change in the probability of the outcome per one unit change in the predictor (Long and Freese 2014). Average marginal effects have the advantage of easy interpretation and comparability across nested logistic regression models. We used the KHB method to estimate a final set of models that calculates the significance of total, direct, and indirect effects of each key predictor (Karlson, Holm, and Breen 2012). Specifically, we used it to test the significance of the indirect effects of race/ethnicity and SES on parental distress through the child's clinical profile and parental beliefs. $^{2}$

We estimated all models using sampling weights and robust standard errors. The weighted estimates are representative of the non-institutionalized population of U.S. children ages 6-17 with special health care needs who have ever been diagnosed with an autism spectrum disorder.

\section{RESULTS}

Before turning to the models for parental beliefs, we evaluate our claim that children's clinical profiles vary by social background. This claim motivated our decision to control for clinical profiles and distinguishes our analysis from previous analyses of parental beliefs. We present descriptive statistics for the child's clinical profile in Appendix Tables A and B. 
Consistent with prior research, several features of the clinical profile differed by race/ethnicity (Rosenberg et al. 2011). Hispanic children were diagnosed at earlier ages than other children, white children were less likely than other children to have comorbid developmental delays or intellectual disabilities, and white children were more likely to be diagnosed with Asperger's. The child's clinical profile also differed in predictable ways by SES. Children of more highly educated parents were less likely to have co-morbid developmental delays and were more likely to be diagnosed with Asperger's. Children of more affluent parents had fewer functional limitations, were less likely to have co-morbid emotional/behavioral problems, developmental, or intellectual disabilities, and were more likely to have been diagnosed with Asperger's. These results suggest that differences in clinical profiles plausibly account for differences in parental distress.

\section{Beliefs about the nature of the condition}

We hypothesized that parental beliefs about the nature of the child's condition vary by race/ethnicity and SES. Table 2 presents average marginal effects and significance levels from logistic regression models predicting parental beliefs, first with demographic controls and then adding indicators of the child's clinical profile. We tested the significance of differences across all racial/ethnic groups with post hoc comparisons.

Parents of children of other races had a .19 higher probability of seeing their children's condition as a mystery than parents of white children (.22 higher than parents of Hispanic children) which remain significant in the presence of controls for the child's clinical profile. After introducing those controls (although not before), they also had a higher probability of believing the symptoms come and go than other parents $(.11, .15$, and .12 , compared to parents of White, Hispanic, and Black children respectively). Parents of Hispanic and Black children had 
lower probabilities of believing their child's condition is lifelong compared to parents of white children (.14 and .20, respectively), and significantly lower than parents of "other race" children (.16 and .22, respectively), which remained significant after controlling for the child's clinical profile.

\section{$<$ Table 2 about here $>$}

Beliefs about the nature of the child's condition also varied by education and income. The probability of viewing the child's condition as a mystery was .10 lower for parents with more, as compared to less, than a high school education. Parents with the highest incomes had a .09 lower probability of believing that the child's symptoms come and go and a .17 higher probability of believing their child's condition could be prevented or decreased with treatment than parents with the lowest incomes. With the exception of the income difference in beliefs regarding treatment, controlling the child's clinical profile had little effect on the average marginal effects for SES. Estimates from the KHB models confirmed that the indirect effects of race/ethnicity and SES on parental beliefs were not significant.

In sum, consistent with expectation, racial and ethnic minority parents and parents of lower SES felt more uncertain about their child's condition. However, inconsistent with expectation, racial and ethnic minority parents were also less likely to see their child's condition as chronic (i.e., not as lifelong).

The child's clinical profile influenced parents' beliefs independent of social background. Parents of children who were diagnosed at ages 3-5 were less likely than parents of children diagnosed at younger ages to believe that the child's condition is a mystery and that it can be prevented or decreased with treatment. Parents of children diagnosed with comorbid developmental delays were more likely to see the condition as lifelong. Parents of children with 
high levels of emotional and behavioral problems were more likely to report believing that the condition is a mystery and also that it is lifelong. Parents of older children were less likely to believe the child's condition comes and goes, more likely to see their child's condition as lifelong, and less likely to believe that it can be decreased with treatment, and that they have the power to change the condition.

\section{Causal attributions}

Parents' causal attributions also varied by race/ethnicity and SES. Table 3 presents the average marginal effects and significance levels from logistic regression models predicting the two types of causal attributions (genetic, environmental).

<Table 3 about here>

The observed differences in beliefs about the causes of autism based on race/ethnicity and parental SES were largely consistent with prior research. Parents of Hispanic children had a .17 lower probability of affirming genetic causes than parents of white children; the difference remained significant in the presence of controls.

Parents in the highest income group had a lower probability of affirming environmental causes than parents in the lowest income group. Children's emotional/behavioral problems and developmental disabilities contributed to explaining the associations of income with causal attributions although the association remained significant.

As was true for beliefs about the nature of the child's condition, the child's clinical profile influenced beliefs about its causes. Parents of children diagnosed at older ages were more likely to affirm a genetic cause; parents of children diagnosed at ages 3-5 were less likely than parents of children diagnosed at earlier ages to affirm an environmental cause. Parents of children with 
developmental delay had a higher probability of affirming an environmental cause, as did parents whose children had emotional and behavioral problems.

The three environmental attributions target different causes, raising concern that the combined measure we used may mask meaningful associations for specific environmental attributions. We re-estimated the models for each environmental attribution separately. The significant associations reported in Table 3 are driven by the associations of race/ethnicity and SES with accident/injury/illness. Although parents of Hispanic children were more likely to affirm each environmental attribution, race/ethnicity was not significantly associated with in utero or post-birth exposure attributions. In contrast, parents of other race children and parents in all three higher-income categories were less likely to affirm accident/injury/illness as causes. (See online supplement for details.)

In sum, parents of Hispanic children were more skeptical of genetic explanations and parents of children of other races and more affluent parents were less likely to affirm environmental causes, especially accident/illness/injury as an explanation for their child's condition.

\section{Predicting parental distress}

We turn next to the questions of how distress differs by social background and whether parental beliefs explain those differences. Table 4 presents the relevant average marginal effects. We begin with models predicting parental distress from race/ethnicity, socioeconomic status, and demographic controls, add the child's clinical profile, and then add parental beliefs.

Parents of Hispanic children had a higher probability than parents of white, Black, and other race children of being upset by their child's condition $(.21, .20$, and .29 , respectively). Parents in the highest, relative to the lowest, income group had a lower probability of being upset. The 
race/ethnic differences survived the wide-ranging controls in Model 2, but the income difference did not. Perhaps not surprisingly, given the significant association of emotional/behavioral problems with parental distress, those problems accounted for most of the decline in the income effect.

$<$ Table 4 about here>

Although some parental beliefs were associated with distress, they contributed little to explaining structured variation in distress. According to the final column of Table 4, controlling for parental beliefs did not explain the association of race/ethnicity with distress. In fact, the average marginal effects for the indicators of race/ethnicity increased rather than decreased and parents of children of other races now had a significantly lower probability of distress. Based on the KHB models, none of the indirect effects was significant. (See online supplement for details.)

The failure of parental beliefs to contribute meaningfully to the associations with distress results from inconsistencies in the associations of beliefs with race/ethnicity and distress. Among parental beliefs, the belief that the child's condition is a mystery and environmental causation were associated with increased distress. Neither of those beliefs differed between parents of Hispanic and white children. In short, parental beliefs matter for distress but mostly not in ways that help us make sense of sociodemographic variation.

\section{DISCUSSION}

We observed variation in parental beliefs about the nature of the child's condition based on race/ethnicity. Parents of children of other races were more likely to see the child's condition as a mystery and as coming and going. Parents of Black and Hispanic children were less likely to believe that the child's condition is lifelong. These differences held independent of controls for 
the child's clinical profile. Interestingly, Black and Hispanic parental beliefs regarding the chronicity of autism did not translate into lower levels of distress.

We also observed significant differences in beliefs about the causes of the child's condition based on race/ethnicity and socioeconomic position. Consistent with prior research on mental disorders, parents of Hispanic children were less likely than parents of white children to affirm genetic explanations as causes of their child's condition (Bussing et al. 1998; Yeh et al.2004). In addition, parents with higher incomes were less likely to endorse environmental factors as an explanation for their child's condition.

Our analysis could not identify the origins of structured variation in parental beliefs. Contrary to our expectation, the variation was not attributable to variation in clinical profiles. Prior research suggests knowledge, culture, and interactions with providers as potential explanations. Low-income parents report less knowledge about autism and have fewer sources of information and fewer contacts with other parents (Mackintosh, Myers, and Goin-Kochel 2005; Pickard and Ingersoll 2016). Culture, vaguely defined, has also been offered as an explanation for subgroup differences in the definition and treatment of autism (Mandell and Novak 2005). Although less often discussed, we suspect that interactions with teachers and providers may differ by race/ethnicity and socioeconomic status in ways that matter for parental beliefs. For example, Cuccaro and colleagues (1996) present evidence that providers see cultural deprivation as a more viable explanation for child problems in low SES families and may, therefore, offer more ambiguous interpretations and advice to low SES parents.

In our sample, parental distress varied systematically by race/ethnicity and SES. Parents of Hispanic children reported being more upset by their child's condition than other parents and parents with higher income reported being less upset. The finding for ethnicity mirrors previous 
findings (Magaña and Smith 2006) and was not explained by ethnic differences in clinical profiles or parental beliefs. Magaña and Smith (2006) hypothesize that higher levels of distress for Latina mothers reflect their greater caregiving responsibilities for family members other than the child, a possibility that we could not evaluate here. The association of income with distress is consistent with specific evidence that economic support reduces stress among parents of children with autism (Falk et al. 2014) and with general evidence that socioeconomic resources are associated with better mental health among parents of chronically ill children (e.g., Ryan et al. 2013). This association was largely explained by subgroup differences in children's clinical profiles, especially the relatively high rates of emotional and behavioral problems among lowerincome children.

Among indicators of the clinical profile, comorbid emotional and behavioral problems contributed most to explaining income-based differences in parental distress. The association of income with emotional and behavioral problems could be attributable to under-diagnosis of autism among lower-income children and the resultant overrepresentation of low-income children with severe problems in the data set (Fountain, King, and Bearman, 2011; Rosenberg et al. 2009). Alternatively, it could reflect differences in access to appropriate and effective care by income. Although we cannot adjudicate these explanations with certainty, we find the latter explanation more plausible inasmuch as other indicators of the severity of the autism (e.g., functional limitations, intellectual disability) did not contribute to explaining income-based differences in distress.

Beliefs mattered for distress but not in the predicted manner. We hypothesized that parental beliefs about the chronicity and controllability of the child's condition would affect distress but they did not. Instead, what mattered for distress was whether parents believed the condition was 
a mystery and whether it was attributed to an environmental cause. These results suggest that parental uncertainty surrounding the condition and beliefs about whether it could have been prevented are more salient dimensions of stress than chronicity and controllability (Cohen 1995). Indeed, most parents believed that the child's condition was lifelong and few were confident that they had the power to change the condition, suggesting that these are accepted characteristics of autism.

As noteworthy was our finding that parental beliefs mattered for distress independent of children's clinical profiles. Interpreted from a stress process framework, these results suggest that objective features of stressors and their subjective interpretations both influence stress responses. Some indicators of the child's clinical profile were associated with distress but so too were some parental beliefs, albeit independently. Of the two broad sets of factors, the child's clinical profile contributed more to explaining socioeconomic differences in parental distress but neither set of factors significantly explained racial/ethnic differences.

Despite the weak explanatory power of our model, we believe that studies of structured variation in both objective stressors and indicators of meaning hold promise. As our analysis demonstrates, people's structural locations influence not only the objective stressors to which they are exposed but also their interpretations of those stressors. To the extent that interpretations are implicated in distress - and our analysis shows that they are - they deserve attention from sociological stress researchers (Pearlin 1989; McLeod 2012).

Our findings were limited by the data to which we had access. All parents in the sample acknowledged that their child had special health care needs; parents who rejected a medical interpretation would not have participated in the survey because they would not have been eligible for recruitment. This sample restriction limits variation in beliefs and may yield 
conservative estimates of the associations of beliefs with other variables. Some eligible parents could not be located or chose not to participate in the survey for what may have been nonrandom reasons, thereby introducing unknown biases. If, for example, the most distressed parents did not participate in the survey, variation in distress would be restricted and the associations of predictors with distress constrained. In addition, the sample was biased toward families whose parents have higher levels of education, likely—in part—because of differential diagnosis based on SES. Although the sample incorporated reasonable variation by income, the education restriction means that we had limited access to the perspectives of parents with low levels of education.

We were also limited by the available measures. We would have liked to have controlled parental age, but no such measure was available. The measures of beliefs were limited in that they were "endorsements" (affirmations of presented options) rather than "suggestions" (spontaneous presentations of beliefs); the latter are more strongly associated with sociodemographic variables that serve as proxies for cultural variation (Pescosolido and Olafsdottir 2010). The indicators for SES were crude, reducing our ability to observe socioeconomic differences in beliefs and responses. Perhaps most important, the measure of distress was also crude: a single item, more similar to measures of perceived parental stress than to the mental health-oriented measures of distress commonly used by sociologists, and for which evidence of metric equivalence across subgroups is not available. Future research would benefit from more nuanced and comprehensive measurement of social group memberships, parental beliefs, and distress.

Despite these limitations, our analysis provides specific information about the correlates of parental beliefs about autism and the role of those beliefs in shaping parental responses based on 
the best available data. In addition, it offers general lessons about the relevance of interpretations of potentially stressful circumstances and causal attributions for stress responses. 


\section{NOTES}

1. Specific items were: "Has it been not true, somewhat true, or certainly true for [name] during the past six months that...[he/she] is generally well behaved, usually does what adults request; [he/she] has many worries, or often seems worried; [he/she] is often unhappy, depressed, or tearful; [he/she] gets along better with adults than with other children/youth; [he/she] has good attention span, sees chores or homework through to the end." Individual items were scored from 0 ("not true") to 2 ("very true") of the child. Total scores range from 0-10, with scores of 6-10 coded as "high."

2. We treated indicators of the child's clinical profile as confounding variables (i.e., we could estimate the degree to which they explained the associations of our key predictors with outcomes) and the child's gender and age as concomitant variables (i.e., controls for which we did not estimate confounding effects). 


\section{REFERENCES}

Al Anbar, Nebal N, Roland M. Dardennes, Arthur Prado-Netto, Kelley Kay, and Yves Contejean. 2010. “Treatment Choices in Autism Spectrum Disorder: The Role of Parental Illness Perceptions." Research in Developmental Disabilities 31:817-28.

Benson, Paul R. 2010. "Coping, Distress, and Well-being in Mothers of Children with Autism." Research in Autism Spectrum Disorders 4(2):217-228.

Bishop, Somer L., Jennifer Richler, Albert C. Cain, and Catherine Lord. 2007. "Predictors of Perceived Negative Impact in Mothers of Children with Autism Spectrum Disorder." American Journal on Mental Retardation 112(6):450-61.

Blacher, J., S. R. Cohen, and G. Azad. 2014. "In the Eye of the Beholder: Reports of Autism Symptoms by Anglo and Latino Mothers." Research in Autism Spectrum Disorders 8:164856.

Blaxton, Jessica M. and C. S. Bergeman. 2017. “A Process-Oriented Perspective Examining the Relationships Among Daily Coping, Stress, and Affect." Personality and Individual Differences 104:357-61.

Bramlett, Matthew D., Stephen J. Blumberg, A. Elizabeth Ormson, Jacquelyn M. George, Kim L. Williams, Alicia M. Frasier, Benjamin, J. Skalland, Kathleen B. Santos, Danielle M.

Vsetecka, Heather M. Morrison, Steven Pedlow, and Fang Wang. 2014. "Design and Operation of the National Survey of Children with Special Health Care Needs, 2009-2010.” Vital Health Statistics 57:1-271.

Bussing, Regina, Nancy E. Schoenberg, and Amy R. Perwien. 1998. "Knowledge and Information about ADHD: Evidence of Cultural Differences among African-American and White Parents." Social Science and Medicine 46(7):919-28. 
Centers for Disease Control and Prevention, National Center for Health Statistics. 2012. State and Local Area Integrated Telephone Survey. 2009-2010 National Survey of CSHCN Frequently Asked Questions: Missing Data and Multiple Imputation. Available from URL: https://www.cdc.gov/nchs/data/slaits/mi_faqs_032112_reformatted_final.pdf.

Chen, Edith, David A. Langer, Yvonne E. Raphaelson, and Karen A. Matthews. 2004. "Socioeconomic Status and Health in Adolescents: The Role of Stress Interpretations." Child Development 75(4):1039-52.

Cohen. Marsha H. 1995. "The Triggers of Heightened Parental Uncertainty in Chronic, LifeThreatening Childhood Illness." Qualitative Health Research 5(1):63-77.

Cohen, Sheldon, T. Kamarck, and Robin Mermelstein. 1983. "A Global Measure of Perceived Stress.” Journal of Health and Social Behavior 24:385-96.

Cohen, Sheldon, Ronald C. Kessler, and Lynn Underwood Gordon. 1997. "Strategies for Measuring Stress in Studies of Psychiatric and Physical Disorders.” Pp. 3-28 in Measuring Stress, edited by S. Cohen, R. C. Kessler, and L. U. Gordon. New York: Oxford.

Cuccaro, Michael L., Harry H. Wright, Christina V. Rownd, Ruth K. Abramson, Jennifer Waller, and Don Gender, 1996. "Brief Report: Professional Perceptions of Children with Developmental Difficulties: The Influence of Race and Socioeconomic Status.” Journal of Autism and Developmental Disorders 26(4):461-9.

Dale, Emily, Andrew Jahoda, and Fiona Knott. 2006. "Mothers' Attributions Following their Child's Diagnosis of Autism Spectrum Disorder." Autism 10(5):463-79.

Daniels, Amy M. and David S. Mandell. 2014. "Explaining Differences in Age at Autism Spectrum Disorder Diagnosis: A Critical Review.” Autism 18(5):583-97. 
DePape, Anne-Marie and Sally Lindsay. 2015. 'Parents' Experiences of Caring for a Child with Autism Spectrum Disorder.” Qualitative Health Research 25(4):569-83.

Falk, Nicholas Henry, Kimberley Norris, and Michael G. Quinn. 2014. "The Factors Predicting Stress, Anxiety and Depression in the Parents of Children with Autism." Journal of Autism and Developmental Disorders 44:3185-203.

Folkman, Susan, Richard S. Lazarus, Christine Dunkel-Schetter, Anita DeLongis, and Rand J. Gruen. 1996. "Dynamics of a Stressful Encounter: Cognitive Appraisal, Coping, and Encounter Outcomes.” Journal of Personality and Social Psychology 50:992-1003.

Foody, Ciara, Jack E. James, and Geraldine Leader. 2014. “Parenting Stress, Salivary Biomarkers, and Ambulatory Blood Pressure in Mothers of Children with Autism Spectrum Disorders." Research in Autism Spectrum Disorders 8(2):99-110.

Fountain, Christine, Marissa D. King, and Peter S. Bearman. 2011. “Age of Diagnosis for Autism: Individual and Community Factors Across 10 Birth Cohorts.” Journal of Epidemiology and Community Health 65(6):503-10.

Francis, Ara. 2013. "Normal Problems or Problem Children? Parents and the Micro-Politics of Deviance and Disability." Pp. 85-96 in Family Troubles? Exploring Changes and Challenges in the Family Lives of Children and Young People, edited by J.R. McCarthy, C. Hooper, and V. Gillies. Bristol, England: Policy Press.

Gannon, Linda and Lynn Pardie. 1989. "The Importance of Chronicity and Controllability of Stress in the Context of Stress-Illness Relationships." Journal of Behavioral Medicine 12(4):357-72. 
Goin-Kochel, Robin P. and Barbara J. Myers. 2005. “Congenital Versus Regressive Onset of Autism Spectrum Disorders.” Focus on Autism and Other Developmental Disabilities 20(3):169-79.

Goin-Kochel, Robin P., Barbara J. Myers, and Virginia H. Mackintosh. 2007. "Parental Reports on the Use of Treatments and Therapies for Children with Autism Spectrum Disorders." Research in Autism Spectrum Disorders 1:195-29.

Goodman, Robert. 1997. “The Strengths and Difficulties Questionnaire: A Research Note.” The Journal of Child Psychology and Psychiatry 38(5):581-6.

Hartley, Sigan L., Emily M. Schaidle, and Cynthia F. Burnson. 2013. "Parental Attributions for the Behavior Problems of Children and Adolescents with Autism Spectrum Disorders.” Journal of Developmental and Behavioral Pediatrics 34:651-60.

Hebert, Elizabeth Baltus and Christina Koulouglioti. 2010. "Parental Beliefs about Cause and Course of their Child's Autism and Outcomes of their Beliefs: A Review of the Literature." Issues in Comprehensive Pediatric Nursing 33:149-63.

Ingersoll, Brooke and David Z. Hambrick. 2011. "The Relationship between the Broader Autism Phenotype, Child Severity, and Stress and Depression in Parents of Children with Autism Spectrum Disorders." Research in Autism Spectrum Disorders 5(1):337-44.

Karlson, Kristian Bernt, Anders Holm, and Richard Breen. 2012. “Comparing Regression Coefficients Between Sample-sample Nested Models Using Logit and Probit.” Sociological Methodology 42(1):286-313.

Kimura, Miyako and Yoshihiko Yamazaki. 2016. "Mental Health and Positive Change among Japanese Mothers of Children with Intellectual Disabilities: Roles of Sense of Coherence and Social Capital." Research in Developmental Disabilities 59:43-54. 
Kuhn, Jennifer C. and Alice S. Carter. 2006. "Maternal Self-Efficacy and Associated Parenting Cognitions among Mothers of Children with Autism." American Journal of Orthopsychiatry 76(4):564-75.

Lazarus, Richard S. and Susan Folkman. 1984. Stress, Appraisal, and Coping. Springer.

Long, J. Scott and Jeremy Freese. 2014. Regression Models for Categorical Dependent Variables Using Stata, Third Edition. College Station, TX: Stata Press.

Mackintosh, Virginia H., Barbara J. Myers, and Robin P. Goin-Kochel. 2005. "Sources of Information and Support Used by Parents of Children with Autism Spectrum Disorders.” Journal on Developmental Disabilities 12(1):41-51.

Magaña, Sandra and Mathew J. Smith. 2006. "Health Outcomes of Midlife and Older Latina and Black American Mothers of Children with Developmental Disabilities." Mental Retardation 44(3):224-34.

Mandell, David S. and Maytali Novak. 2005. "The Role of Culture in Families' Treatment Decisions for Children with Autism Spectrum Disorders." Mental Retardation and Developmental Disabilities Research Reviews 11(2):110-5.

McLeod, Jane D. 2012. “The Meanings of Stress.” Society and Mental Health 2(3):172-86. McStay, Rebecca L., Cheryl Dissanayake, Anke Scheeren, Hans M. Koot, and Sander Begeer. 2014. "Parenting Stress and Autism: The Role of Age, Autism Severity, Quality of Life and Problem Behaviour of Children and Adolescents with Autism.” Autism: 18(5):502-10.

Mirowsky, John and Catherine E. Ross. 1986. "Social Patterns of Distress." Annual Review of Sociology 12:23-45. 
Monat, Alan, James R. Averill, and Richard S. Lazarus. 1972. “Anticipatory Stress and Coping Reactions Under Various Conditions of Uncertainty.” Journal of Personality and Social Psychology 24(2):237-53.

Mullins, Alexandria, J., Kaitlyn L. Gamwell, Christina M. Sharkey, Dana M. Bakula, Alayna P. Tackett, Kristina I. Suorsa, John M. Chaney, and Larry L. Mullins. 2017. “Illness Uncertainty and Illness Intrusiveness as Predictors of Depressive and Anxious Symptomology in College Students with Chronic Illnesses.” Journal of American College Health 65(5):352-60.

National Research Council. 2001. Educating Children with Autism. Committee on Educational Interventions for Children with Autism. Catherine Lord and James P. McGee, eds. Division of Behavioral and Social Sciences and Education. Washington, DC: National Academy Press.

Newschaffer, Craig J., Lisa A. Croen, Julie Daniels, Ellen Giarelli, Judith K. Grether, Susan E. Levy, David S. Mandell, Lisa A. Miller, Jennifer Pinto-Martin, Judy Reaven, Ann M. Reynolds, Catherine E. Rice, Diana Schendel, and Gayle C. Windham. 2007. "The Epidemiology of Autism Spectrum Disorders." Annual Review of Public Health 28:235-58. Pearlin, Leonard I. 1989. "The Sociological Study of Stress.” Journal of Health and Social Behavior 30:241-56.

Pescosolido, Bernice A. and Sigrun Olafsdottir. 2010. "The Cultural Turn in Sociology: Can It Help Us Resolve an Age-Old Problem in Understanding Decision Making for Health Care?" Sociological Forum 25(4):655-76.

Phelan, Jo C. 2005. "Geneticization of Deviant Behavior and Consequences for Stigma: The Case of Mental Illness.” Journal of Health and Social Behavior 46(4):307-22. 
Pickard, Katherine E. and Brooke R. Ingersoll. 2016. “Quality versus Quantity: The Role of Socioeconomic Status on Parent-Reported Service Knowledge, Service Use, Unmet Service Needs, and Barriers to Service Use.” Autism 20(1):106-15.

Rosenberg, Rebecca E., Amy M. Daniels, J. Kiely Law, Paul A. Law, and Walter E. Kaufmann. 2009. “Trends in Autism Spectrum Disorder Diagnoses: 1994-2007.” Journal of Autism \& Developmental Disorders 39(8):1099-1111.

Rosenberg, Rebecca E., Walter E. Kaufmann, J. Kiely Law, and Paul A. Law. 2011. "Parent Report of Community Psychiatric Comorbid Diagnoses in Autism Spectrum Disorders.” Autism Research and Treatment 2011:1-10.

Ryan, Jamie L., Angelica R. Eddington, Stephanie E. Hullman, Rachelle R. Ramsey, Cortney Wolfe-Christensen, John M. Chaney, and Larry L. Mullins. 2013. “An Examination of Parenting Capacity Variables and Child Adjustment Outcomes Across Socioeconomic Level in Pediatric Cancer." Children's Health Care 42:281-93.

Santacroce, Sheila Judge. 2003. "Parental Uncertainty and Posttraumatic Stress in Serious Childhood Illness." Journal of Nursing Scholarship 35(1):45-51.

Serrata, Cynthia A. 2012. "Psychosocial Aspects of Parenting a Child with Autism." Journal of Applied Rehabilitation Counseling 43(4):29-35.

Simon, Robin W. 1995. “Gender, Multiple Roles, Role Meaning, and Mental Health.” Journal of Health and Social Behavior 36:182-94.

Singh, Jennifer S. 2016. Multiple Autisms: Spectrums of Advocacy and Genomic Science. Minneapolis, MN: University of Minnesota Press.

Thoits, Peggy A. 1991. “On Merging Identity Theory and Stress Research.” Social Psychology Quarterly 54:101-12. 
Thoits, Peggy A. 1995. "Stress, Coping, and Social Support Processes: Where Are We? What Next?" Journal of Health and Social Behavior Special Issue:53-79.

Turner, R. Jay, Blair Wheaton, and Donald Lloyd. 1995. "The Epidemiology of Social Stress." American Sociological Review 60:104-25.

Weiner, Bernard. 1985. "An Attributional Theory of Achievement Motivation and Emotion." Psychological Review 92:548-73.

Wheaton, Blair. 1990. "Life Transitions, Role Histories, and Mental Health.” American Sociological Review 55:209-23.

Yeh, May, Richard L. Hough, Kristen McCabe, Anna Lau, and Ann Garland. 2004. "Parental Beliefs about the Causes of Child Problems: Exploring Racial/Ethnic Patterns.” Journal of the American Academy of Child and Adolescent Psychiatry 43(5):605-12.

Zuckerman, Katharine E., Brianna Sinche, Martiza Cobian, Marlene Cervantes, Angie Mejia, Thomas Becker, and Christina Nicolaidis. 2014. "Conceptualization of Autism in the Latino Community and its Relationship with Early Diagnosis." Journal of Developmental and Behavioral Pediatrics 35:522-33.

Zuckerman, Katharine Elizabeth, Olivia Jasmine Lindly, Brianna Kathleen Sinche, and Christina Nicolaidis. 2015. "Parent Health Beliefs, Social Determinants of Health, and Child Health Services Utilization Among US School-Age Children with Autism.” Journal of Developmental and Behavioral Pediatrics 36(3):146-57.

Zuckerman, Katharine E., Olivia J. Lindly, and Brianna Sinche. 2016. "Parent Beliefs about the Causes of Learning and Developmental Problems Among Children With Autism Spectrum Disorder: Results from a National Survey.” American Journal on Intellectual and Developmental Disabilities 121(5):432-47. 
Table 1. Descriptive Statistics

\section{Parental beliefs}

Nature of condition

Is a mystery $(\mathrm{N}=1370)$

Definitely agree

$15.99 \%$

Somewhat agree

$26.42 \%$

Definitely/somewhat disagree

Symptoms come and go $(\mathrm{N}=1377)$

Definitely agree

$57.59 \%$

Somewhat agree

$8.64 \%$

$21.06 \%$

Definitely/somewhat disagree

$70.30 \%$

Lifelong $(\mathrm{N}=1374)$

Definitely agree

$74.31 \%$

Somewhat agree

$20.31 \%$

Definitely/somewhat disagree

$5.39 \%$

Can be treated $(\mathrm{N}=1362)$

Definitely agree

Somewhat agree

Definitely/somewhat disagree

$36.05 \%$

$44.27 \%$

$19.68 \%$

Have power to change $(\mathrm{N}=1367)$

Definitely agree

$10.10 \%$

$32.33 \%$

Somewhat agree

$57.57 \%$

Causal attributions

Genetic $(\mathrm{N}=1304)$

Definitely agree

$30.67 \%$

$37.88 \%$

Somewhat agree

$31.44 \%$

Any environmental attribution $(\mathrm{N}=1378)$

Definitely agree to any

$33.24 \%$

Parental distress $(\mathrm{N}=1271)$

Definitely agree

$23.98 \%$

$32.19 \%$

Somewhat agree

$43.82 \%$

Social background

Race/ethnicity

White

$75.04 \%$

Hispanic

$8.42 \%$

$6.31 \%$

Black

$10.23 \%$

Other race

$86.07 \%$

Parent education $>$ HS

$14.01 \%$

Up to $99 \%$ of FPL

$19.45 \%$

100-199\% of FPL

$33.31 \%$ 
$>=400 \%$ of FPL

$33.24 \%$

\section{Clinical profile}

Age diagnosed with autism

$0-2$ years

$17.49 \%$

3-5 years

$39.91 \%$

6-17 years

$42.60 \%$

Developmental delay

$64.51 \%$

Intellectual disability

$21.19 \%$

Autism diagnosis

Pervasive developmental disorder (PDD) $\quad 11.97 \%$

Autistic disorder

$17.71 \%$

Asperger's

$18.65 \%$

Multiple diagnoses

$42.67 \%$

Unknown

$9.00 \%$

Emotional/behavioral problems

$39.04 \%$

Functional limitations

$64.37 \%$

Male

$81.42 \%$

Note: Sample sizes for dependent variables are given in parentheses. 
Table 2. Average Marginal Effects from Logistic Regression Models for the Nature of the Condition

\begin{tabular}{|c|c|c|c|c|c|c|c|c|}
\hline & \multicolumn{2}{|c|}{$\begin{array}{l}\text { Is mystery } \\
(\mathrm{N}=1370)\end{array}$} & \multicolumn{2}{|c|}{$\begin{array}{l}\text { Comes and goes } \\
\quad(\mathrm{N}=1377)\end{array}$} & \multicolumn{2}{|c|}{$\begin{array}{c}\text { Lifelong } \\
(\mathrm{N}=1374)\end{array}$} & \multicolumn{2}{|c|}{$\begin{array}{c}\text { Can be treate } \\
(\mathrm{N}=1362)\end{array}$} \\
\hline & Model 1 & Model 2 & Model 1 & Model 2 & Model 1 & Model 2 & Model 1 & Mod \\
\hline \multicolumn{9}{|l|}{ Social Background } \\
\hline \multicolumn{9}{|l|}{ Race/ethnicity } \\
\hline Hispanic & -.03 & -.05 & -.04 & -.04 & $-.14 *$ & $-.16^{*}$ & .00 & -.03 \\
\hline Black & .04 & .04 & -.02 & -.01 & $-.20^{*}$ & $-.20 * *$ & .12 & .10 \\
\hline Other race & $.19 * *$ & $.17 * *$ & .10 & $.11 *$ & .02 & -.01 & .01 & -.01 \\
\hline Parent education $>\mathrm{HS}$ & $-.10 *$ & $-.12 *$ & -.03 & -.04 & -.05 & -.01 & .02 & .00 \\
\hline \multicolumn{9}{|l|}{ Household income } \\
\hline $100-199 \%$ of FPL & -.05 & -.03 & -.05 & -.04 & -.01 & .02 & .01 & .00 \\
\hline $200-399 \%$ of FPL & -.04 & -.02 & $-.08 *$ & -.07 & -.00 & .06 & .07 & .05 \\
\hline$>=400 \%$ of FPL & -.04 & -.01 & $-.09 *$ & $-.08 *$ & -.03 & .05 & $.17 *$ & .14 \\
\hline \multirow{2}{*}{\multicolumn{9}{|c|}{$\begin{array}{l}\text { Clinical Profile } \\
\text { Age diagnosed with } \\
\text { autism }\end{array}$}} \\
\hline & & & & & & & & \\
\hline Age 3-5 & & $-.12 *$ & & .00 & & .01 & & -.18 \\
\hline Age 6-17 & & -.10 & & .01 & & .04 & & -.09 \\
\hline Developmental delay & & -.04 & & -.04 & & $.10^{*}$ & & -.06 \\
\hline Intellectual disability & & .05 & & .03 & & .03 & & .05 \\
\hline \multicolumn{9}{|l|}{ Autism diagnosis } \\
\hline PDD & & -.01 & & -.06 & & .01 & & .08 \\
\hline Autistic disorder & & .11 & & -.03 & & .03 & & .05 \\
\hline Multiple diagnoses & & .03 & & -.00 & & .05 & & .14 \\
\hline Unknown & & -.05 & & -.01 & & .06 & & .06 \\
\hline $\begin{array}{l}\text { Emotional/behavioral } \\
\text { problems }\end{array}$ & & $.07 *$ & & .04 & & $.15^{* * *}$ & & -.04 \\
\hline Functional limitations & & .01 & & -.04 & & $.14 * *$ & & -.05 \\
\hline \multicolumn{9}{|l|}{ Controls } \\
\hline Male & .01 & .01 & -.04 & -.04 & .03 & -.01 & -.08 & -.07 \\
\hline Age & -.00 & -.00 & $-.01 *$ & -.01 & $.04 * * *$ & $.03 * *$ & $-.03 * * *$ & -.03 \\
\hline
\end{tabular}


Table 3. Average Marginal Effects from Logistic Regression Models for Parents' Causal Attributions

\begin{tabular}{|c|c|c|c|c|}
\hline \multirow[b]{3}{*}{ Independent Variables } & \multicolumn{2}{|c|}{$\begin{array}{l}\text { Genetic } \\
(\mathrm{N}=1304)\end{array}$} & \multicolumn{2}{|c|}{$\begin{array}{c}\text { Environmental } \\
\quad(\mathrm{N}=1378)\end{array}$} \\
\hline & Model 1 & Model 2 & Model 1 & Model 2 \\
\hline & & & & \\
\hline \multicolumn{5}{|l|}{ Race/ethnicity } \\
\hline Hispanic & $-.17 * *$ & $-.16 * *$ & .13 & .08 \\
\hline Black & -.04 & -.04 & .13 & .12 \\
\hline Other race & -.03 & -.01 & -.04 & -.07 \\
\hline Parent education $>$ HS & -.05 & -.04 & -.11 & -.08 \\
\hline \multicolumn{5}{|l|}{ Household income } \\
\hline $100-199 \%$ of FPL & .04 & .03 & -.09 & -.06 \\
\hline $200-399 \%$ of FPL & -.02 & .01 & -.15 & -.11 \\
\hline$>=400 \%$ of FPL & -.02 & -.01 & $-.20 *$ & $-.14 *$ \\
\hline \multicolumn{5}{|l|}{ Clinical Profile } \\
\hline \multicolumn{5}{|l|}{ Age diagnosed with autism } \\
\hline Age $3-5$ & & $.13 * *$ & & $-.13 *$ \\
\hline Age 6-17 & & $.15^{* *}$ & & -.03 \\
\hline Developmental delay & & .01 & & $.13 * *$ \\
\hline Intellectual disability & & -.00 & & .07 \\
\hline \multicolumn{5}{|l|}{ Autism diagnosis } \\
\hline PDD & & -.07 & & .06 \\
\hline Autistic disorder & & $-.18 *$ & & .12 \\
\hline Multiple diagnoses & & .01 & & .02 \\
\hline Unknown & & .10 & & .03 \\
\hline Emotional/behavioral problems & & .08 & & $.10 *$ \\
\hline Functional limitations & & .06 & & .02 \\
\hline \multicolumn{5}{|l|}{ Controls } \\
\hline Male & $.13^{*}$ & .10 & .02 & -.01 \\
\hline Age & -.01 & -.01 & .01 & .00 \\
\hline
\end{tabular}


Table 4. Average Marginal Effects from Logistic Regression Models for Distress

\section{Independent Variables}

Race/ethnicity

Hispanic

Black

Other race

Parent education $>$ HS

Household income

$100-199 \%$ of FPL

$200-399 \%$ of FPL

$>=400 \%$ of FPL

\section{Clinical profile}

Age diagnosed with autism

Age 3-5

Age 6-17

Developmental delay

Intellectual disability

Autism diagnosis

PDD

Autistic disorder

Multiple diagnoses

Unknown

Emotional/behavioral

problems

Functional limitations

\section{Parental Beliefs}

Nature of condition

Is a mystery

Symptoms come and go

Model 1

Model 2

Model 3

Lifelong

$.21 * *$

.00

$-.08$

$-.11$

$-.03$

$-.03$

$-.15^{*}$
$.21 * *$

.01

$-.09$

$-.09$

$-.02$

.01

$-.08$
$.22 * *$

$-.01$

$-.10 *$

$-.05$

.01

.03

$-.06$

Can be treated

$.03 \quad .06$

$.01 \quad .01$

$.05 \quad .03$

$.02-.00$

$\begin{array}{ll}-.00 & -.02 \\ .02 & -.01\end{array}$

$.02-.01$

$.10 \quad .07$

$.03 \quad .02$

$.19 * * * \quad .16^{* * *}$

.04

.03

Have power to change

Causal attributions

Genetic

Environmental

$.21 * *$

$-.07$

.03

.01

$-.05$

.02

$.15 * * *$

Controls

Male

.08

$-.00$

.04

.04

Age

$-.00$

$-.01$

Notes: $\mathrm{N}=1,271 ;{ }^{*} \mathrm{p}<.05,{ }^{*} \mathrm{p}<.01$. Reference categories are race/ethnicity: white, income: up to $99 \%$ FPL, age diagnosed: 0-2, and diagnosis: Asperger's. 
Table S1. Average Marginal Effects from Logistic Regression Models for Environmental Attributions

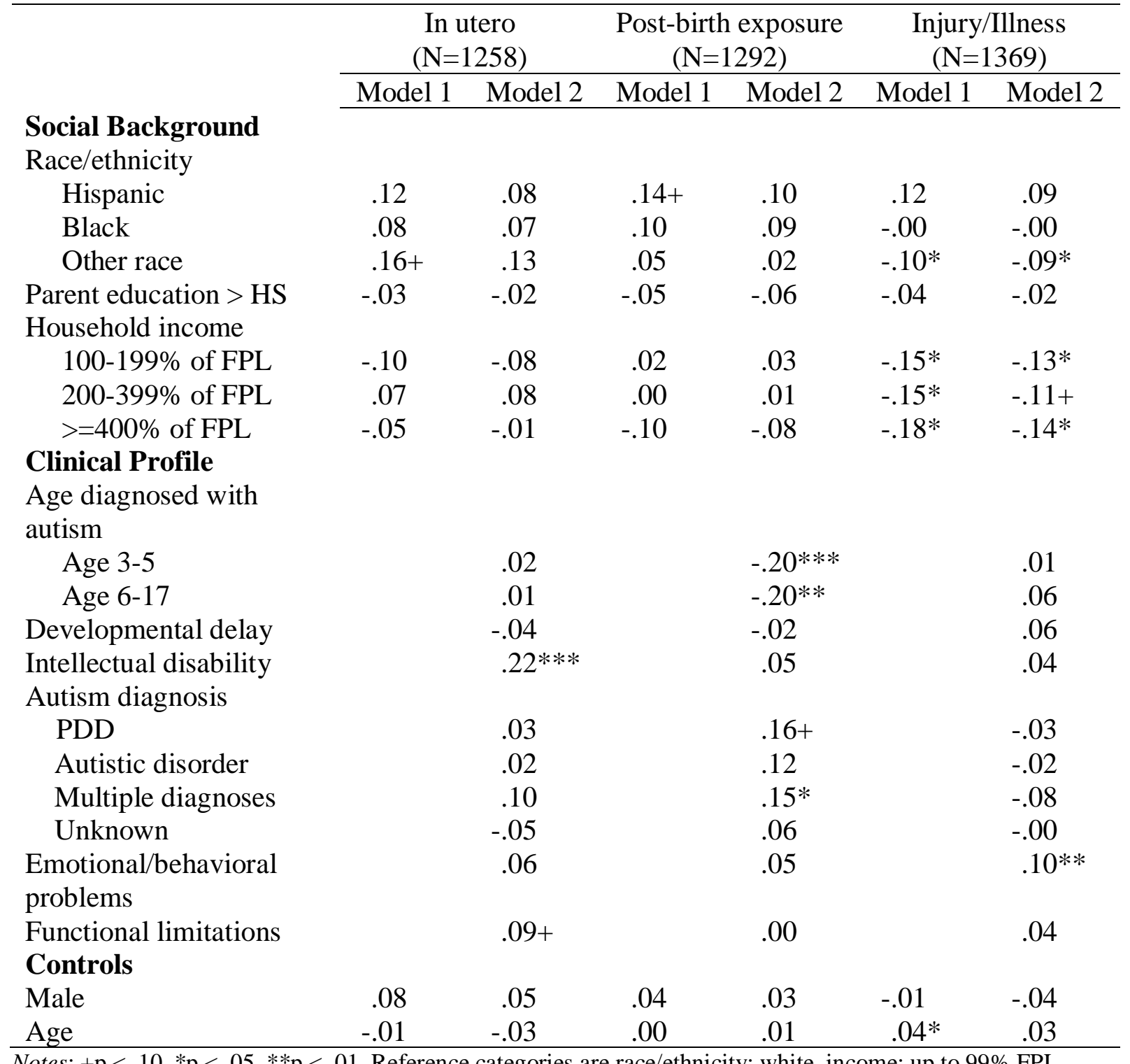

Notes: $+\mathrm{p}<.10, * \mathrm{p}<.05, * * \mathrm{p}<.01$. Reference categories are race/ethnicity: white, income: up to $99 \%$ FPL, age diagnosed: 0-2, and diagnosis: Asperger's. 
Table S2. Decomposition of Effects of Primary Independent Variables Using the KHB Method

\begin{tabular}{|c|c|c|c|c|}
\hline Variable & Coeff & $\mathrm{SE}$ & $\mathrm{Z}$ & $\mathrm{P}>|\mathrm{z}|$ \\
\hline \multicolumn{5}{|l|}{ Hispanic } \\
\hline Reduced & 1.16 & .36 & 3.26 & .00 \\
\hline Full & 1.22 & .36 & 3.40 & .00 \\
\hline Difference & -.06 & .36 & -.16 & .87 \\
\hline \multicolumn{5}{|l|}{ Black } \\
\hline Reduced & .00 & .37 & .01 & .99 \\
\hline Full & -.09 & .38 & -.23 & .82 \\
\hline Difference & .09 & .36 & .25 & .81 \\
\hline \multicolumn{5}{|l|}{ Other Race } \\
\hline Reduced & -.69 & .41 & -1.70 & .09 \\
\hline Full & -.79 & .42 & -1.90 & .06 \\
\hline Difference & .09 & .36 & .26 & .79 \\
\hline \multicolumn{5}{|l|}{$\begin{array}{l}\text { Parental } \\
\text { education }\end{array}$} \\
\hline Reduced & -.54 & .31 & -1.74 & .08 \\
\hline Full & -.32 & .31 & -1.02 & .31 \\
\hline Difference & -.22 & .36 & -.62 & .54 \\
\hline \multicolumn{5}{|l|}{ Household } \\
\hline \multicolumn{5}{|l|}{ Income } \\
\hline \multicolumn{5}{|c|}{$100-199 \%$ of } \\
\hline \multicolumn{5}{|l|}{ FPL } \\
\hline Reduced & -.09 & .34 & -.24 & .81 \\
\hline Full & .03 & .35 & .09 & .93 \\
\hline Difference & -.12 & .36 & -.32 & .75 \\
\hline \multicolumn{5}{|c|}{$\begin{array}{l}200-399 \% \text { of } \\
\text { FPL }\end{array}$} \\
\hline Reduced & .13 & .35 & .37 & .71 \\
\hline Full & .20 & .34 & .58 & .56 \\
\hline Difference & -.07 & .36 & -.20 & .84 \\
\hline \multicolumn{5}{|c|}{$>=400 \%$ of FPL } \\
\hline Reduced & -.52 & .37 & -1.41 & .16 \\
\hline Full & -.39 & .37 & -1.07 & .29 \\
\hline Difference & -.13 & .36 & -.35 & .73 \\
\hline
\end{tabular}

Notes: The tables lists coefficients, standard errors, z-statistics, and p-values from KHB decomposition models. The "reduced" model refers to the model without explanatory variables. The "full" model includes all variables. The "difference" represents the indirect effect. 